\title{
Assessment of Cardiovascular Risk indices in Type 2 Diabetes Mellitus
}

\author{
Adu EM ${ }^{1 *}$, Ukwamedu $\mathrm{HA}^{1}$ and Oghagbon ES ${ }^{2}$ \\ ${ }^{1}$ Department of Medical Laboratory Services, Antiretroviral Therapy Centre, Central Hospital Agbor, Delta State, Nigeria \\ ${ }^{2}$ Department of Medical Biochemistry, School of Basic Medical Sciences, College of Medical Sciences, University of Benin, Benin City, Edo State, Nigeria \\ *Corresponding author: Adu EM, Department of Medical Laboratory Services, Antiretroviral Therapy Centre, Central Hospital Agbor, Delta State, Nigeria, Tel: \\ +2348034258067; E-mail: adumatthew10@yahoo.com
}

Received date: April 08, 2015, Accepted date: April 24, 2015, Published date: April 30, 2015

Copyright: @ 2015 Adu EM et al. This is an open-access article distributed under the terms of the Creative Commons Attribution License, which permits unrestricted use, distribution, and reproduction in any medium, provided the original author and source are credited.

\begin{abstract}
Background: Dyslipidemia a common feature of diabetes mellitus leads to cardiovascular complications. These complications are not detected early enough due to absence of cheap and routine biomarker.

Aim: Therefore, the aim of this study is to assess the cardiovascular risk indices of diabetes mellitus individuals using Atherogenic coefficient (Ac), Cardiac Risk Ratio (CRR), Atherogenic index of plasma (AIP) and Non- HDL cholesterol (surrogate marker for apolipoprotein B) in this locality.

Methods: Serum Total Cholesterol (TC), Triglycerides (TG), High Density Lipoprotein Cholesterol (HDL-C), Low Density Lipoprotein-Cholesterol (LDL-C), Very Low Density Lipoprotein-Cholesterol (VLDL-C) as well as cardiovascular risk indices (Cardiac Risk Ratio (CRR), atherogenic coefficient (Ac), atherogenic index of plasma (AIP) and Non-HDL cholesterol) were assessed.

Results: The TC,TG,LDL-C,VLDL-C as well as all the cardiometabolic risk indices of diabetes were observed to be significantly high $(P<0.05)$ when compared with control subjects. HDL-C in diabetes was observed to be significantly low $(P<0.05)$ when compared with control subjects.

Conclusion: The results indicates greater propensity of diabetes to cardiovascular complications. We therefore advocate routine use of these indices as part of lipid profile in order to nip any cardiovascular complication early enough.
\end{abstract}

Keywords: Diabetes mellitus; Cardiovascular risk indices; Dyslipidemia; Biomarker; Atherogenic

\section{Introduction}

Diabetes Mellitus (DM) is a metabolic disorder characterized by hyperglyceamia resulting from a variable interaction of hereditary and environmental factors due to defects in insulin secretion, insulin inaction, or both (WHO, 1985). There are two types of DM (Type 1 and Type 2) depending on the availability or non-responses of the insulin. Type $2 \mathrm{DM}$ makes up about $90 \%$ of the diabetic population in Nigeria [1]. Relevant abnormalities found in diabetes mellitus are chronic hyperglycemia, dyslipidemia and insulin resistance [2]. Previous authors have reported increased levels of total cholesterol, triglycerides as well as LDL-cholesterol but decreased levels of HDLcholesterol in diabetes $[1,2]$. Dyslipidaemia is common in diabetes, as both insulin deficiency and insulin resistance affects enzymes and pathways of lipid metabolism. It has been observed that about seventyeight percent of type 2 diabetic patients die from cardiovascular disease, due to premature atherosclerosis which involves dyslipidemia [3]. Many factors account for increased cardiovascular disease risk in diabetes but lipid abnormalities are major contributors. It is well known that people with type 2 diabetes have elevated cardiovascular risk. Adults with diabetes have a two to four fold higher risk of experiencing cardiovascular events than adults without diabetes $[4,5]$. Due to higher risk of diabetes mellitus to cardiovascular disease, it is crucial to identify and address these cardiovascular risks. The measurement of LDL cholesterol alone does not provide sufficient measure of atherogenic risk in hypertriglyceridemic patients, and a second or several markers is warranted [6]. Literature showed that the association of LDL-C with CVD was weak and LDL-C was not a significant predictor of CHD mortality [7]. Although there are recent markers such as apolipoproteins, C-reactive Proteins and markers of insulin resistance in cardiovascular risk assessment still present but are expensive. Also these recent markers are not routinely examined to assess the patient vulnerability to cardiovascular diseases. Therefore, the aim of this study is to assess the cardiovascular risk of diabetes mellitus individuals using atherogenic coefficient (Ac), Cardiac Risk Ratio (CRR), Atherogenic index of plasma (AIP) and Non- HDL cholesterol a surrogate marker for apolipoprotien B in this locality [8].

\section{Materials and Methods}

\section{Study population}

A total of Two hundred (200) participants were recruited for this study based on the prevalence of diabetes in this locality. This comprise of One hundred (100) Non- Insulin Dependent Diabetes Mellitus (NIDDM) and One hundred (100) non diabetes individuals as controls. Inclusion criteria include being diabetic while an exclusion criterion is non-diabetic, cigarette smoking and lipid lowering drugs. Routine urinalysis was also performed for participants to confirm 
Page 2 of 4

diabetes and rule out renal or liver pathology. Informed consent was obtained from participants as well as ethical clearance from the various institutional ethics committee.

\section{Collection of samples}

Fasting blood samples were collected by standard vein puncture into plain containers. The blood was allowed to clot and then centrifuged at $3000 \mathrm{rpm}$ for 10 minutes. The serum was separated into a cryovail tubes and kept frozen until required for analysis.

\section{Biochemical analysis}

Serum Total Cholesterol and Triglycerides were analyzed using the enzymatic CHOD-PAP method of as modified by and HDLCholesterol analyzed by the method of Trinder as modified by Richmond and HDL-Cholesterol analyzed by the method of Burstein [9-11]. LDL-Cholesterol, VLDL-Cholesterol was calculated using equation Friedewald except for those that have triglycerides values of $\geq$ $400 \mathrm{mg} / \mathrm{dl}$ which were estimated directly [12]. Cardiac Risk Ratio (CRR) was calculated as TC/HDL, atherogenic coefficient (Ac) was calculated as TC-HDL/HDL, and atherogenic index of plasma (AIP) as $\log$ TG/HDL $[13,14]$. Non-HDL cholesterol was calculated as TC-HDL [8]. All test kits used were commercially available and products of Randox Laboratories UK. In all analysis, manufacturer's instructions were adhered to strictly.

\section{Statistical analysis}

The groups mean \pm SD was calculated for each analyte and significant difference between means evaluated using the student $\mathrm{t}$ test. Statistical Package for Social Science SPSS version 16.0 software (SPSS Inc., Chicago, IL USA) for windows was used, with $\mathrm{P}<0.05$ considered as statistically significant.

\section{Results}

The results of our study shows an increase in total cholesterol, triglycerides, VLDL and LDL cholesterol but decreased level of HDL Cholesterol in Diabetes subjects when compared with controls (Table 1 and Figure 1).

\begin{tabular}{|l|l|l|l|l|l|l|l|l|}
\hline & Age (years) & BMI & FBS (mmol/l) & TC (mmol/l) & TG (mmol/l) & HDL (mmol/l) & LDL (mmol/l) & VLDL (mmol/l) \\
\hline NIDDM & $47.3 \pm 0.7$ & $26.4 \pm 0.2$ & $11.3 \pm 0.4$ & $5.7 \pm 0.3$ & $3.2 \pm 0.1$ & $1.4 \pm 0.1$ & $2.8 \pm 0.1$ & $1.5 \pm 0.1$ \\
\hline Controls & $30.6 \pm 0.7$ & $22.1 \pm 0.3$ & $4.7 \pm 0.1$ & $3.8 \pm 0.3$ & $1.3 \pm 0.1$ & $2.0 \pm 0.1$ & $1.2 \pm 0.1$ & $0.6 \pm 0.1$ \\
\hline P value & $\mathrm{P}=16.7<0.05$ & $\mathrm{P}=-43<0.05$ & $\mathrm{P}=22<0.05$ & $\mathrm{P}=1.9<0.05$ & $\mathrm{P}=1.9<0.05$ & $\mathrm{P}=-0.6<0.05$ & $\mathrm{P}=1.6<0.05$ & $\mathrm{P}=0.6<0.05$ \\
\hline
\end{tabular}

Table 1: Lipid profile (mmol/l) of Diabetic subjects and Controls.

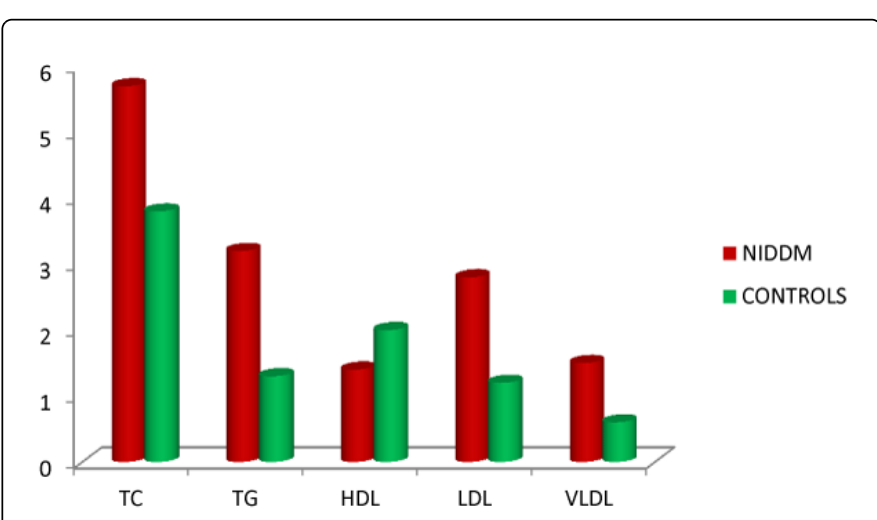

Figure 1: Serum Lipid Profile of Diabetes and Control compared.

\begin{tabular}{|l|l|l|l|l|}
\hline \multicolumn{1}{|c|}{ CRR } & Ac & AIP & Non HDL \\
\hline NIDDM & $4.1 \pm 3.0$ & $3.1 \pm 2.0$ & $0.4 \pm 0.0$ & $4.3 \pm 0.2$ \\
\hline Controls & $1.9 \pm 3.0$ & $0.9 \pm 2.0$ & $-0.2 \pm 0.0$ & $1.8 \pm 0.2$ \\
\hline P value & $\mathrm{P}=2.2<0.05$ & $\mathrm{P}=2.2<0.05$ & $\mathrm{P}=0.6<0.05$ & $\mathrm{P}=2.5<0.05$ \\
\hline
\end{tabular}

Table 2: Cardiovascular Disease Risk Indices of Diabetic and Control Subjects.

Table 2 shows increased cardiovascular risk indices in diabetes when compared with control subjects.

\section{Discussion}

Lipid tests, including triglycerides (TG), total cholesterol (TC), high density lipoprotein cholesterol (HDL-C), and low density lipoprotein cholesterol (LDL-C), are well accepted and widely used for the assessment of cardiovascular disease (CVD) and its equivalents, including peripheral vascular disease, abdominal aortic aneurysm, and ischemic cerebral vascular disease [6]. The results of this study show statistically significant increased total cholesterol, triglycerides, LDLcholesterol and VLDL-cholesterol but decreased HDL-cholesterol in diabetes patients when compared with control subjects. This is in tandem with the work of Bello -Sani et al, Onyemelukwe and Stafford, and Rasha who did similar work on diabetes $[1,15,16]$.

The Low Density Lipoprotein -Cholesterol (LDL-C) has been a prime index for assessing CVD but the measurement of LDL cholesterol alone does not provide sufficient measure of atherogenic risk in hypertriglyceridemic patients and second or other biomarkers is warranted. Literature showed that the association of LDL-C with CVD was weak and LDL-C was not a significant predictor of Coronary Heart Disease (CHD) mortality [6,7].

This study observed a statistically significant increased level of Cardiac risk ratio (CRR) in diabetes mellitus $(4.1 \pm 3.0)$ when compared with controls $(1.9 \pm 3.0)$. This is in accordance with the work of VinodMahato et al and Swaminathan et al who obtained a value of $4.3 \pm 0.13$ and $4.5 \pm 2.5$ for diabetes respectively $[17,18]$. When CRR of diabetes and controls were compared with the value of American Heart Association $(\leq 3.5)$, the Diabetes shows greater propensity towards cardiovascular disease. 
The Atherogenic Coefficient $(A c)$ of diabetes $(3.1 \pm 2.0)$ in our study was observed to be significantly increased when compared with the non- diabetes subjects $(0.9 \pm 2.0)$. This is in agreement with the work of Ikewuchi, who obtain Ac of 2.04 in diabetic rats. The observed value of 3.1 in diabetes shows a greater tendency towards cardiovascular disease [19].

The Atherogenic Index of Plasma (AIP) is a mathematical relationship between TG and HDL-C and an additional index for assessing cardiovascular (CV) risk factors [20]. The association of TGs and HDL-C in this simple ratio reflects the balance between risk and protective lipoprotein forces. The AIP of diabetes in this study was observed to be significantly increased $(0.3 \pm 0.0)$ when compared with the control $(-0.2 \pm 0.0)$. This is in accordance with Sushith et al., [21] who observed 0.24 in diabetic smokers and 0.49 diabetic nonsmokers. It has been suggested that AIP values of -0.3 to 0.1 are associated with low, 0.1 to 0.24 with medium and $>0.24$ with high Cardiovascular risk [22]. Sushith and his coworkers [21] concluded that AIP has a better prediction of Coronary Artery Disease than triglycerides or High Density lipoprotein alone. This is because AIP is positively correlated with the fractional esterification rate of HDL (FERHDL), and also inversely correlated with LDL particle size. FERHDL predict particle size in HDL and LDL, which in turn predicts the risk of coronary heart disease. The simultaneous use of TG and HDL as AIP is useful in predicting plasma atherogenicity. This ratio has a strong predictor of myocardial infarction [23]. Atherogenic Index of Plasma provides information about the atherogenicity of plasma and quantifies the response to therapeutic intervention [14]. Also AIP correlate inversely with insulin sensitivity measurement [14].

Non HDL-C is calculated as total cholesterol minus HDL-C. It is a single index of all the Atherogenic, apolipoprotein (apo) B- containing lipoproteins, Low Density Lipoprotein, Very Low Density Lipoprotein, Intermediate Density Lipoprotein and lipoprotein (a). Although apo B can be measured directly, measurement of Non HDL cholesterol is more practical, reliable and inexpensive and is accepted as a surrogate marker for apoB in routine clinical practice [8]. The result of our study shows a significantly increased Non-HDL-C in Diabetes Mellitus patients $(4.3 \pm 0.2)$ when compared with non-diabetes $(1.2 \pm 0.2)$. The cut-off value for non HDL-c is $130 \mathrm{mg} / \mathrm{dl}(3.3 \mathrm{mmol} / \mathrm{l})$ which shows that Diabetes Mellitus patients has a higher risk of cardiovascular disease [8]. Non-HDL-C has been observed to have the strongest relationship with small dense LDL-C (sdLDL-C) levels when compared with other lipid measurement [24]. The predictive value of non-HDL-C for cardiovascular risk and mortality is better than that of LDL-C [25]. The strong association between non-HDL-C and sdLDL$\mathrm{C}$ adds additional support for using the non-HDL-C level as a predictor of CVD mortality [24].

\section{Conclusion}

In conclusion, it is worthy of note that these cardiovascular risk indices which are not routinely included in lipid profile are good and better biomarkers than the LDL-C alone in the assessment of cardiovascular risk in patients that are prone to cardiovascular diseases such as diabetes mellitus. We therefore advocate the inclusion of these cardiovascular risk indices in the routine lipid profile for a better assessment of the patient.

\section{Source of Funding: None}

Conflict of Interest: None declared
Acknowledgement: We wish to acknowledge our participants who agree to take part in this survey.

\section{References}

1. Bello - Sani F, Bakari AG and Anumah FE (2007) Dyslipidaemia in persons with type 2 diabetes mellitus in Kaduna, Nigeria. Int J Diab Metab 15: 9-13.

2. Reaven GM, Bernstein R, Davis B, Olefsky JM (1976) Non-ketotic diabetes mellitus; insulin deficiency or insulin resistance? American Journal Medicine, 60: 80-88.

3. Eschwe'ge E (2003) The dysmetabolic syndrome, insulin resistance, and increase in cardiovascular mortality and morbidity in type 2 diabetes: etiological factors in the development of cardiovascular complications. Diabetes Metab 29: 6519-6527.

4. Laakso M (2001) Cardiovascular disease in type 2 diabetes: challenge for treatment and prevention. J Intern Med 249: 225-235.

5. Fox CS, Coady S, Sorlie PD, Levy D, Meigs JB, et al. (2004) Trends in cardiovascular complications of diabetes. JAMA 292: 2495-2499.

6. National Cholesterol Education Program (NCEP) (2002) Expert Panel on Detection, Evaluation, and Treatment of High Blood Cholesterol in Adults (ATP 111): Third Report of the NCEP 106:3143-3421.

7. Lehto S, Ronnemaa T, Pyorala K, Laakso M (2000) Cardiovascular risk factors clustering with endogenous hyperinsulinaemia predict death from coronary heart disease in patients with type 11 diabetes. Diabetologia 43:148-155.

8. Anie LP (2008) Clinical Relevance of Non-HDL Cholesterol in Patients with Diabetes. Clinical Diabetes 26: 3-6.

9. Veiga F, Fernandes C, Teixeira F (2000) Oral bioavailability and hypoglycaemic activity of tolbutamide/cyclodextrin inclusion complexes. Int J Pharm 202: 165-171.

10. Richmond W (1973) Cholesterol enzymatic colorimetric test CHOP PAP method of estimation of total cholesterol in serum. Clin Chem 191: 1350-1356.

11. Burstein M, Scholnick HR, Morfin R (1970) Rapid method for the isolation of lipoproteins from human serum by precipitation with polyanions. J Lipid Res 11: 583-595.

12. Friedewald WT, Levy RI, Fredrickson DS (1972) Estimation of the concentration of low-density lipoprotein cholesterol in plasma, without use of the preparative ultracentrifuge. Clin Chem 18: 499-502.

13. Ikewuchi CJ, Ikewuchi CC (2009) Alteration of Plasma Lipid Profile and Atherogenic indices of Cholesterol loaded Rats by Tridax Procumbens Linn: Implications for the Management of Obesity and Cardiovascular Diseases. Biokemistri 21: 95-99.

14. Dobiásová M (2004) Atherogenic index of plasma [ $\log$ (triglycerides/ HDL-cholesterol)]: theoretical and practical implications. Clin Chem 50: 1113-1115.

15. Onyemelukwe GC, Stafford WL (1981) Serum lipids in Nigerians: the effect of diabetes mellitus. Trop Geogr Med 33: 323-328.

16. Rasha SN (2008) A Study of Serum Lipid Profile in Obese NDDM Patients. J Al-Nahrain University 11: 106-110.

17. VinodMahato R, Gyawali P, Raut PP, Regmi P, Singh K P, et al. (2011) Association between glycaemic control and serum lipid profile in type 2 diabetic patients: Glycated haemoglobin as a dual biomarker. Biomedical Research 22: 375-380.

18. Swaminathan S, Priya G, Revathy K and Emila S (2013) Non HDL-C as an alternate to LDL-C for the diagnosis of Cardio Vascular Disease. Journal Pharmaceutical and Biomedical Sciences 30: 913-919.

19. Ikewuchi CC (2010) Effect of Aqueous Extract of Sansevieria senegambica Baker on Plasma Chemistry, Lipid Profile and Atherogenic Indices of Alloxan Treated Rats: Implications for the Management of Cardiovascular Complications in Diabetes Mellitus. Pacific Journal of Science and Technology 11: 524-531.

20. Dobiásová M, Frohlich J (2001) The plasma parameter log (TG/HDL-C) as an atherogenic index: correlation with lipoprotein particle size and 
Citation: Adu EM, Ukwamedu HA, Oghagbon ES (2015) Assessment of Cardiovascular Risk indices in Type 2 Diabetes Mellitus. Trop Med Surg 3: 184. doi:10.4172/2329-9088.1000184

Page 4 of 4

esterification rate in apoB-lipoprotein-depleted plasma (FER(HDL)) Clin Biochem 34: 583-588.

21. 21. Sushith, Shetty J K, Jeppu A K, Bhagavath P, Shipa S K, Rashmi M, Kamath V and Shetty S (2012) Lipid profile and Atherogenic Index of Plasma (AIP) in diabetes non-smokers and smokers in coastal Karnataka. Int J AJ Institute Med Sci 1: 93-97.

22. Dobiásová M (2006) [AIP--atherogenic index of plasma as a significant predictor of cardiovascular risk: from research to practice]. Vnitr Lek 52 64-71.
23. Gaziano JM, Hennekens CH, O'Donnell CJ, Breslow JL, Buring JE (1997) Fasting triglycerides, high-density lipoprotein, and risk of myocardial infarction. Circulation 96: 2520-2525.

24. Srisawasdi P, Chaloeysup S, Teerajetgul Y, Pocathikorn A, Sukasem C, et al. (2011) Estimation of plasma small dense LDL cholesterol from classic lipid measures. Am J Clin Pathol 136: 20-29.

25. Miller M, Ginsberg HN, Schaefer EJ (2008) Relative atherogenicity and predictive value of non-high-density lipoprotein cholesterol for coronary heart disease. Am J Cardiol 101: 1003-1008. 